\title{
An expanded mixed finite element simulation for two-sided time-dependent fractional diffusion problem
}

\section{Qiong Yuan and Huanzhen Chen ${ }^{*}$}

\section{"Correspondence:}

chhzh@sdnu.edu.cn

School of Mathematics and

Statistics, Shandong Normal

University, Jinan, China

\section{严 Springer}

\begin{abstract}
In this paper, we consider a time-dependent diffusion problem with two-sided Riemann-Liouville fractional derivatives. By introducing a fractional-order flux as auxiliary variable, we establish the saddle-point variational formulation, based on which we employ a locally conservative mixed finite element method to approximate the unknown function, its derivative and the fractional flux in space and use the backward Euler scheme to discrete the time derivative, and thus propose a fully discrete expanded mixed finite element procedure. We prove the well-posedness and the optimal order error estimates of the proposed procedure for a sufficiently smooth solution. Numerical experiments are presented to confirm our theoretical findings.
\end{abstract}

Keywords: time-dependent fractional diffusion problem; expanded mixed finite element method; fully discrete scheme; well-posedness; error estimates

\section{Introduction}

We consider the following time-dependent fractional diffusion equation of order $2-\beta$ :
(a) $\frac{\partial u}{\partial t}-D\left\{K\left(\theta_{0} I_{x}^{\beta}+(1-\theta)_{x} I_{1}^{\beta}\right) D u\right\}(x, t)=f(x, t), \quad x \in \Omega, t \in(0, T]$,
(b) $u(x, 0)=u^{0}, \quad x \in \bar{\Omega}$,
(c) $u(0, t)=u(1, t)=0, \quad t \in[0, T]$,

where $\Omega=(0,1), 0<\beta<1,0 \leq \theta \leq 1, K$ is the diffusivity coefficient and $f \in L^{2}(\Omega)$ is the source or sink term; $D=\frac{\partial}{\partial x}$ is the first-order derivative operator, ${ }_{0} I_{x}^{\beta}$ and ${ }_{x} I_{1}^{\beta}$ represent the left and right fractional integral operators of order $\beta$, respectively, defined by (2.1).

The interest in (1.1) is motivated by its application to physical phenomena. Numerous experiments show that fractional diffusion equations have more advantages and higher accuracy in modeling anomalous or non-Fickian diffusion processes that arise from turbulent flow [1, 2], chaotic dynamics [3] and viscoelasticity [1]. Recently, a series of definition of the fractional derivative were proposed [4-6]. These new definitions can better describe the chemical kinetics system pertaining [7], the generation of nonlinear waterwaves in the long-wavelength regime [8], the convective straight fins with temperaturedependent thermal conductivity [9], the relaxation and diffusion models [4], optimal con-

(c) The Author(s) 2018. This article is distributed under the terms of the Creative Commons Attribution 4.0 International License (http://creativecommons.org/licenses/by/4.0/), which permits unrestricted use, distribution, and reproduction in any medium, provided you give appropriate credit to the original author(s) and the source, provide a link to the Creative Commons license, and indicate if changes were made. 
trol problems [5], the motion of a bead sliding on a wire [10], the material heterogeneities and structures with different scales [6].

In general, Fourier transform and Laplace transform are useful tools to obtain the analytic solutions of fractional partial differential equations. However, these two methods are available only in a few limited cases. Thus, it is important to find practical numerical means to deal with fractional model. In the last decade, different numerical methods have been developed, such as the difference method [11, 12], the spectral method [13], the fast difference method [14], the finite volume method [15, 16], the homotopy analysis transform method $[17,18]$, the efficient nonstandard finite difference method [19], the Riesz-Caputo difference method [20], and the Riesz-Riemann-Liouville difference method [21].

Galerkin finite element method is another way to solve fractional derivative equations. In the series of works [22-24], Ervin and Roop presented a first rigorous analysis for the stationary fractional advection dispersion equation based on a variational formulation. Then the discontinuous Galerkin method [25], mixed finite element method [26-30], Petrov Galerkin method [31] and the least-squared mixed method are proposed [32] for stationary fractional diffusion equations, consecutively.

In this article, we employ an expanded mixed finite element to discrete fractional diffusion part and use the backward Euler scheme to approximate the time derivative, and thus propose a fully discrete procedure for the time-dependent fractional diffusion equation (1.1). The solvability and stability of the fully discrete scheme is proved and the optimalorder numerical analysis are presented. Numerical experiments are conducted to verify our theoretical findings.

This paper is organized as follows. In Section 2, we recall preliminaries on fractional calculus and present the equivalent relations among negative fractional derivative spaces, fractional derivative spaces and the standard Sobolev spaces. In Section 3, by introducing the flux function $p=-K\left(\theta_{0} I_{x}^{\beta}+(1-\theta)_{x} I_{1}^{\beta}\right) D u$ and $q=D u$ we derive the corresponding saddle-point formulation and the locally conservative expanded mixed finite element procedure, with its well-posedness analyzed. Optimal-order error analysis for fully regular solution would be given in Section 4 and numerical experiments are performed to verify our theoretical results in Section 5. In Section 6, some concluding remarks are presented.

\section{Preliminaries}

We first briefly revisit the definitions and some properties of left-sided and right-sided Riemann-Liouville fractional derivatives.

Let $\mu>0,{ }_{0} I_{x}^{\mu}$ be the left-sided fractional integral operator of order $\mu$, defined by

$$
{ }_{0} I_{x}^{\mu} u=\frac{1}{\Gamma(\mu)} \int_{0}^{x}(x-s)^{\mu-1} u(s) d s
$$

where $\Gamma(\cdot)$ is the Gamma function. Based on the definition of integral operator, we introduce the left-sided Riemann-Liouville fractional derivative of order $\mu$ in [33-35]. Let $n \in N^{+}$satisfy $n-1<\mu<n$, then

$$
{ }_{0} D_{x}^{\mu} u=\frac{d^{n}}{d x^{n}}\left({ }_{0} I_{x}^{n-\mu} u\right)
$$


Similarly, the right versions of fractional-order integral and derivative are defined as

$$
{ }_{x} I_{1}^{\mu} u=\frac{1}{\Gamma(\mu)} \int_{x}^{1}(s-x)^{\mu-1} u(s) d s
$$

and

$$
{ }_{x} D_{1}^{\mu} u=(-1)^{n} \frac{d^{n}}{d x^{n}}\left({ }_{x} I_{1}^{n-\mu} u\right) .
$$

The fractional integral operator ${ }_{0} I_{x}$ and ${ }_{x} I_{1}$ satisfy the semigroup property

$$
\begin{aligned}
& { }_{0} I_{x}^{v+\mu} u={ }_{0} I_{x}^{v} 0 I_{x}^{\mu} u, \\
& { }_{x} I_{1}^{v+\mu} u={ }_{x} I_{1}^{v} I_{1}^{\mu} u, \quad \text { for all } u \in L^{2}(\Omega)
\end{aligned}
$$

and the adjoint property

$$
\left({ }_{0} I_{x}^{\mu} u, v\right)=\left(u,{ }_{x} I_{1}^{\mu} v\right), \quad \text { for all } u, v \in L^{2}(\Omega)
$$

The left fractional derivative spaces $J_{L, 0}^{\mu}(\Omega)[23]$ are defined as the closure of $C_{0}^{\infty}(\Omega)$ under the norms $\|\cdot\|_{J_{L}^{\mu}}$

$$
\begin{aligned}
& |u|_{J_{L}^{\mu}(\Omega)}:=\left\|_{0} D_{x}^{\mu} u\right\|_{L^{2}(\Omega)}, \\
& \|u\|_{J_{L}^{\mu}(\Omega)}:=\left(\|u\|_{L^{2}(\Omega)}^{2}+|u|_{J_{L}^{\mu}(\Omega)}^{2}\right)^{1 / 2} .
\end{aligned}
$$

The right fractional derivative spaces $J_{R, 0}^{\mu}(\Omega)$ are defined similarly. In these spaces, the fractional differential operators satisfy the semigroup property [23]

$$
{ }_{0} D_{x}^{\mu} u={ }_{0} D_{x 0}^{v} D_{x}^{\mu-v} u, \quad \text { for all } u \in J_{L, 0}^{\mu}(\Omega) .
$$

The equivalence theory for the fractional derivative spaces is described by the following lemma.

Lemma 2.1 ([23], Theorem 2.13) Let $\mu>0$ and $\mu \neq n-\frac{1}{2}, n=1,2, \ldots$. Then the $J_{L, 0}^{\mu}(\Omega)$ and $J_{R, 0}^{\mu}(\Omega)$ are equal to the fractional-order Sobolev space $H_{0}^{\mu}(\Omega)$, with equivalent semi-norms and norms.

Parallel to Lemma 2.1, we shall define the negative fractional derivative spaces and establish their equivalence theory with the negative fractional-order Sobolev spaces.

The left negative fractional derivative spaces $J_{L}^{-\mu}(\Omega)$ [36] are defined as the closure of $C_{0}^{\infty}(\Omega)$ under the norms $\|\cdot\|_{L}^{-\mu}$

$$
\|u\|_{L}^{-\mu}(\Omega):=\left\|I_{x}^{\mu} u\right\|_{L^{2}(\Omega)} .
$$

The right negative fractional derivative spaces $J_{R}^{-\mu}(\Omega)$ are defined similarly. 
Lemma 2.2 ([36], Theorem 2.6) Let $\mu>0, \mu \neq n-\frac{1}{2}, n=1,2,3, \ldots$. Then the negative fractional spaces $J_{L}^{-\mu}(\Omega)$ and $J_{R}^{-\mu}(\Omega)$ are equal to the standard negative fractional Sobolev space $H^{-\mu}(\Omega)$ with equivalent norms. Furthermore,

$$
\left({ }_{0} I_{x}^{\mu} v,{ }_{x} I_{1}^{\mu} v\right)=\cos (\mu \pi)\|v\|_{H^{-\mu}(\Omega)}^{2} .
$$

For simplicity, we only use $\|\cdot\|_{\mu},|\cdot|_{\mu}$ or $\|\cdot\|_{-\mu}$ to represent their norms and semi-norms in the following sections. When $\mu=0$, we understand $H^{0}(\Omega)=L^{2}(\Omega)$ and simply use $\|\cdot\|$ to denote its norm.

We also need the definition of Sobolev spaces involving time defined by, for any Banach space $X$,

$$
\begin{aligned}
& W_{q}^{m}\left(t_{1}, t_{2} ; X\right):=\left\{f(x, t):\left\|\frac{\partial^{\alpha} f}{\partial t^{\alpha}}(\cdot, t)\right\|_{X} \in L^{q}\left(t_{1}, t_{2}\right), 0 \leq \alpha \leq m, 1 \leq q<\infty\right\}, \\
& \|f\|_{W_{q}^{m}\left(t_{1}, t_{2} ; X\right)}:= \begin{cases}\left(\sum_{\alpha=0}^{m} \int_{t_{1}}^{t_{2}}\left\|\frac{\partial^{\alpha} f}{\partial t^{\alpha}}(\cdot, t)\right\|_{X}^{q} d t\right)^{\frac{1}{q}}, & 1 \leq q<\infty, \\
\max _{0 \leq \alpha \leq m} \operatorname{esssup}_{t \in\left(t_{1}, t_{2}\right)}\left\|\frac{\partial^{\alpha} f}{\partial t^{\alpha}}(\cdot, t)\right\|_{X}, & q=\infty .\end{cases}
\end{aligned}
$$

We conclude this section by the following commonly used inequality.

Lemma 2.3 (Discrete Gronwall inequality) Let $\triangle t, B, C>0,\left(a_{n}\right)_{n},\left(b_{n}\right)_{n},\left(c_{n}\right)_{n},\left(d_{n}\right)_{n}$ be sequence of nonnegative numbers satisfying

$$
a_{n}+\triangle t \sum_{i=0}^{n} b_{i} \leq B+C \triangle t \sum_{i=0}^{n} a_{i}+\triangle t \sum_{i=0}^{n} c_{i}, \quad \forall n \geq 0
$$

Then, if $C \triangle t<1$, we have

$$
a_{n}+\Delta t \sum_{i=0}^{n} b_{i} \leq e^{C(n+1) \Delta t}\left(B+\Delta t \sum_{i=0}^{n} c_{i}\right), \quad \forall n \geq 0
$$

\section{Mixed finite element procedure}

We begin this section by introducing the intermediate variable $q, p$ and rewrite (1.1) into the following saddle-point problem:
(a) $q=D u, \quad x \in \Omega$,
(b) $p=-K\left(\theta_{0} I_{x}^{\beta}+(1-\theta)_{x} I_{1}^{\beta}\right) q, \quad x \in \Omega$,
(c) $u_{t}+D p=f, \quad x \in \Omega$,
(d) $u(x, 0)=u^{0}$.

We let $V:=H^{1}(\Omega), H:=H^{-\frac{\beta}{2}}(\Omega)$ and $W:=L^{2}(\Omega)$. In order to define the variational formulations, we multiply (3.1a) by any $v \in V$ and integrate by parts over $\Omega$. We multiply (3.1b) by any $\sigma \in H$ and (3.1c) by any $w \in W$, then integrate over $\Omega$. Combining the homogeneous boundary condition, we define the saddle-point variational formulation corresponding to 
(3.1) or (1.1) as to find $(p, q, u) \in V \times H \times W$ such that
(a) $(q, v)+(u, D v)=0, \quad \forall v \in V$,
(b) $(p, \sigma)+\left(K\left(\theta_{0} I_{x}^{\beta}+(1-\theta)_{x} I_{1}^{\beta}\right) q, \sigma\right)=0, \quad \forall \sigma \in H$,
(c) $\left(u_{t}, w\right)+(D p, w)=(f, w), \quad \forall w \in W$,
(d) $u(x, 0)=u^{0}$.

Based on the saddle-variational formulations (3.2), we shall construct a fully discrete mixed finite element scheme for the fractional diffusion equation(1.1).

Let us uniformly divide $\Omega=[0,1]$ by $I_{i}=\left[x_{i-1}, x_{i}\right], i=1,2, \ldots, M$ with $x_{0}=0, x_{M}=1$, $h=x_{i}-x_{i-1}$. Let $t_{n}=n \tau, n=0,1, \ldots, J$, where $\tau=T / J$ is time step. We denote RaviartThomas spaces or Brézzi-Douglas-Marini spaces [28, 37-39] by $V_{h} \times W_{h} \subset V \times W$ with space index $k \geq 0$ and $H_{h} \subset H$ is a piecewise finite dimensional subspace defined by

$$
\begin{aligned}
& V_{h}=\left\{v_{h} \in V ;\left.v_{h}\right|_{I_{i}} \in P_{k+1}\left(I_{i}\right), k \geq 0\right\}, \\
& H_{h}=\left\{\sigma_{h} \in H ;\left.\sigma_{h}\right|_{I_{i}} \in P_{k+1}\left(I_{i}\right), k \geq 0\right\}, \\
& W_{h}=\left\{w_{h} \in W ;\left.w_{h}\right|_{I_{i}} \in P_{k}\left(I_{i}\right), k \geq 0\right\} .
\end{aligned}
$$

Here $P_{k}\left(I_{i}\right)$ is the restriction of all polynomials of degree not bigger than $k$ to $I_{i}$.

We use the backward Euler scheme to discrete the time derivative of first order and define the fully discrete mixed finite element procedure of (3.2) so as to find $\left(p_{h}^{n}, q_{h}^{n}, u_{h}^{n}\right) \in$ $V_{h} \times H_{h} \times W_{h}$ such that
(a) $\left(q_{h}^{n}, v_{h}\right)+\left(u_{h}^{n}, D v_{h}\right)=0, \quad \forall v_{h} \in V_{h}$,
(b) $\left(p_{h}^{n}, \sigma_{h}\right)+\left(K\left(\theta_{0} I_{x}^{\beta}+(1-\theta)_{x} I_{1}^{\beta}\right) q_{h}^{n}, \sigma_{h}\right)=0, \quad \forall \sigma_{h} \in H_{h}$,
(c) $\left(\frac{u_{h}^{n}-u_{h}^{n-1}}{\tau}, w_{h}\right)+\left(D p_{h}^{n}, w_{h}\right)=\left(f^{n}, w_{h}\right), \quad \forall w_{h} \in W_{h}$,
(d) $u_{h}^{0}=R_{h} u^{0}$.

We assume

$$
V_{h}=\operatorname{span}\left\{\varphi_{i}\right\}_{i=1}^{N+1}, \quad H_{h}=\operatorname{span}\left\{\varphi_{j}\right\}_{j=1}^{N+1}, \quad W_{h}=\operatorname{span}\left\{\phi_{k}\right\}_{k=1}^{L},
$$

and express $p_{h}^{n}, q_{h}^{n}, u_{h}^{n}$ as

$$
p_{h}^{n}=\sum_{i=1}^{N+1} p_{i}^{n} \varphi_{i}, \quad q_{h}^{n}=\sum_{j=1}^{N+1} q_{j}^{n} \varphi_{j}, \quad u_{h}^{n}=\sum_{k=1}^{L} u_{k}^{n} \phi_{k} .
$$

Substituting the expression of $p_{h}^{n}, q_{h}^{n}, u_{h}^{n}$ into (3.4) to rewrite it into a standard algebraic equation

$$
\left\{\begin{array}{l}
\text { (a) } E Q^{n}+B^{T} U^{n}=0, \\
\text { (b) } A Q^{n}+E P^{n}=0, \\
\text { (c) } \tau B P^{n}+H U^{n}=F^{n},
\end{array}\right.
$$


and into its matrix form

$$
\left[\begin{array}{ccc}
E & 0 & B^{T} \\
A & E & 0 \\
0 & \tau B & H
\end{array}\right]\left[\begin{array}{c}
Q^{n} \\
P^{n} \\
U^{n}
\end{array}\right]=\left[\begin{array}{c}
0 \\
0 \\
F^{n}
\end{array}\right],
$$

where

$$
\begin{aligned}
& P=\left(p_{1}^{n}, p_{2}^{n}, \ldots, p_{N+1}^{n}\right)^{T}, \quad Q=\left(q_{1}^{n}, q_{2}^{n}, \ldots, q_{N+1}^{n}\right)^{T}, \quad U=\left(u_{1}^{n}, u_{2}^{n}, \ldots, u_{L}^{n}\right)^{T}, \\
& H=\left(\left(\phi_{k}, \phi_{m}\right)\right)_{L \times L}, \quad B=\left(\left(D \varphi_{i}, \phi_{k}\right)\right)_{L \times(N+1)}, \quad E=\left(\left(\varphi_{i}, \varphi_{j}\right)\right)_{(N+1) \times(N+1)}, \\
& A=\left(\left(K\left(\theta_{0} I_{x}^{\beta}+(1-\theta)_{x} I_{1}^{\beta}\right) \varphi_{j}, \varphi_{i}\right)\right)_{(N+1) \times(N+1)^{\prime}} \\
& F^{n}=\tau\left[\left(f^{n}, \phi_{1}\right),\left(f^{n}, \phi_{2}\right), \ldots,\left(f^{n}, \phi_{L}\right)\right]^{T}+H U^{n-1} .
\end{aligned}
$$

Remark 3.1 In non-Fickian diffusion processes, $p=-K\left({ }_{0} I_{x}^{\beta}+{ }_{x} I_{1}^{\beta}\right) D u$ is the fractional diffusive flux and thus $p_{h}^{n}$ can be understood as the numerical fractional diffusive flux. By taking $W_{h}=1$ in (3.4)(c), we get

$$
\int_{I_{i}} \frac{u_{h}^{n}}{\tau}+p_{h}^{n}\left(x_{i}\right)-p_{h}^{n}\left(x_{i-1}\right)=\left(f^{n}, 1\right)+\int_{I_{i}} \frac{u_{h}^{n-1}}{\tau}, \quad \forall 1 \leq n \leq J,
$$

which implies that the mass is preserved element by element. In fact, the left hand side of (3.7) is the the mass accumulated over the interval $I_{i}$ at $t=t_{n}$ per unit time and the spreading through the boundary of the interval $I_{i}$, the right hand side of (3.7) is the mass accumulated over the interval $I_{i}$ at $t=t_{n-1}$ and from the source term, and the $=$ sign refers to the conservation element by element. That is, the fully discrete expanded mixed finite element scheme is conservative locally.

In the following discussion, we shall present the solvability and stability for the proposed fully discrete expanded mixed finite element scheme (3.4).

Theorem 3.1 There exists a unique solution $U^{n}, P^{n}, Q^{n}$ to (3.6) for any $n=1,2, \ldots, J$ and $\tau>0$.

Proof We begin to show that the matrix $A$ is positive definite. Notice the semi-group property (2.5), the adjoint property (2.6), the definition of $J_{L}^{-\mu}$, Lemma 2.2 and (2.8) to derive

$$
\begin{aligned}
(A \sigma, \sigma) & =\left(K\left(\theta_{0} I_{x}^{\beta}+(1-\theta){ }_{x} I_{1}^{\beta}\right) \sigma, \sigma\right) \\
& =K \theta\left({ }_{0} I_{x}^{\frac{\beta}{2}} \sigma,{ }_{x} I_{1}^{\frac{\beta}{2}} \sigma\right)+K(1-\theta)\left({ }_{x} I_{1}^{\frac{\beta}{2}} \sigma,{ }_{0} I_{x}^{\frac{\beta}{2}} \sigma\right) \\
& =K \cos \frac{\beta}{2} \pi\left\|_{0} I_{x}^{\frac{\beta}{2}} \sigma\right\|^{2} \\
& \geq C\|\sigma\|_{-\frac{\beta}{2}}^{2} .
\end{aligned}
$$

This implies that the matrix $A$ is positive definite. 
Noting that $E$ is symmetric positive matrix, we can solve $P^{n}$ from (3.5)(b),

$$
P^{n}=-E^{-1} A Q^{n},
$$

and $Q^{n}$ from (3.5)(a),

$$
Q^{n}=-E^{-1} B^{T} U^{n}
$$

Substituting them into (3.5)(c) to have

$$
\left(H+\tau B\left(E^{-1} A E^{-1}\right) B^{T}\right) U^{n}=F^{n} .
$$

$E^{-1} A E^{-1}$ being positive definite implies that $B\left(E^{-1} A E^{-1}\right) B^{T}$ is positive semi-definite, from which one derives $H+\tau B\left(E^{-1} A E^{-1}\right) B^{T}$ to be positive definite for $\forall \tau>0$ by combining with the positive definiteness of $H$. Hence, there exists a unique $U^{n}$. Substituting $U^{n}$ into (3.9) and (3.8), we can solve the unique $Q^{n}$ and $P^{n}$, which completes the proof.

Theorem 3.2 The fully discrete scheme (3.4) is unconditional stable, that is, for any $\tau>0$ and $h>0$, there exists a constant $C$ such that

$$
\max _{1 \leq n \leq J}\left\|u_{h}^{n}\right\|^{2}+\tau \sum_{n=1}^{J}\left\|q_{h}^{n}\right\|_{-\frac{\beta}{2}}^{2}+\tau \sum_{n=1}^{J}\left\|p_{h}^{n}\right\|^{2} \leq C(T, K, \beta)\left(\left\|u_{h}^{0}\right\|^{2}+\max _{1 \leq n \leq J}\left\|f^{n}\right\|^{2}\right) .
$$

Proof Setting $v_{h}=p_{h}^{n}, \sigma_{h}=q_{h}^{n}$, $w_{h}=u_{h}^{n}$ in the (3.4), we can get
(a) $\left(q_{h}^{n}, p_{h}^{n}\right)+\left(u_{h}^{n}, D p_{h}^{n}\right)=0$,
(b) $\left(p_{h}^{n}, q_{h}^{n}\right)+\left(K\left(\theta_{0} I_{x}^{\beta}+(1-\theta)_{x} I_{1}^{\beta}\right) q_{h}^{n}, q_{h}^{n}\right)=0$,
(c) $\left(\frac{u_{h}^{n}-u_{h}^{n-1}}{\tau}, u_{h}^{n}\right)+\left(D p_{h}^{n}, u_{h}^{n}\right)=\left(f^{n}, u_{h}^{n}\right)$,
(d) $u_{h}^{0}=R_{h} u^{0}$.

From (3.10) we can get

$$
\left(K\left(\theta_{0} I_{x}^{\beta}+(1-\theta)_{x} I_{1}^{\beta}\right) q_{h}^{n}, q_{h}^{n}\right)+\left(\frac{u_{h}^{n}-u_{h}^{n-1}}{\tau}, u_{h}^{n}\right)=\left(f^{n}, u_{h}^{n}\right) .
$$

Due to the fact that

$$
\left(K\left(\theta_{0} I_{x}^{\beta}+(1-\theta)_{x} I_{1}^{\beta}\right) q_{h}^{n}, q_{h}^{n}\right)=K \cos \frac{\beta}{2} \pi\left\|q_{h}^{n}\right\|_{-\frac{\beta}{2}}^{2}
$$

and

$$
\left(u_{h}^{n}-u_{h}^{n-1}, u_{h}^{n}\right) \geq \frac{1}{2}\left(\left\|u_{h}^{n}\right\|^{2}-\left\|u_{h}^{n-1}\right\|^{2}\right)
$$

we can obtain

$$
\begin{aligned}
\left\|u_{h}^{n}\right\|^{2}-\left\|u_{h}^{n-1}\right\|^{2}+\tau\left\|q_{h}^{n}\right\|_{-\frac{\beta}{2}}^{2} & \leq C(K, \beta) \tau\left(f^{n}, u_{h}^{n}\right) \\
& \leq C(K, \beta) \tau\left(\left\|f^{n}\right\|^{2}+\left\|u_{h}^{n}\right\|^{2}\right) .
\end{aligned}
$$


Add all the terms from $n=1$ to $n=J$ to get

$$
\begin{aligned}
\left\|u_{h}^{J}\right\|^{2}-\left\|u_{h}^{0}\right\|^{2}+\tau \sum_{n=1}^{J}\left\|q_{h}^{n}\right\|_{-\frac{\beta}{2}}^{2} & \leq C(K, \beta) \tau\left(\sum_{n=1}^{J}\left\|f^{n}\right\|^{2}+\sum_{n=1}^{J}\left\|u_{h}^{n}\right\|^{2}\right) \\
& \leq C(K, \beta)\left(T \max _{1 \leq n \leq J}\left\|f^{n}\right\|^{2}+\tau \sum_{n=1}^{J}\left\|u_{h}^{n}\right\|^{2}\right) .
\end{aligned}
$$

By using the discrete Gronwall inequality, we have

$$
\left\|u_{h}^{J}\right\|^{2}+\tau \sum_{n=1}^{J}\left\|q_{h}^{n}\right\|_{-\frac{\beta}{2}}^{2} \leq C(K, T, \beta)\left(\left\|u_{h}^{0}\right\|^{2}+\max _{1 \leq n \leq J}\left\|f^{n}\right\|^{2}\right) .
$$

Notice that the spaces $V_{h}$ and $H_{h}$ are the same, set $v_{h}=\sigma_{h}=p_{h}^{n}, w_{h}=u_{h}^{n}$ in (3.4). Then we can obtain

$$
\left(p_{h}^{n}, p_{h}^{n}\right)=-\left(K\left(\theta_{0} I_{x}^{\beta}+(1-\theta)_{x} I_{1}^{\beta}\right) q_{h}^{n}, p_{h}^{n}\right)
$$

so that

$$
\left\|p_{h}^{n}\right\|^{2} \leq C\left\|q_{h}^{n}\right\|_{-\frac{\beta}{2}}\left\|_{0} I_{x}^{\frac{\beta}{2}} p_{h}^{n}\right\|_{\frac{\beta}{2}}
$$

When $0<\beta<1$, the two spaces $H_{0}^{\frac{\beta}{2}}(\Omega)$ and $J_{L}^{\frac{\beta}{2}}(\Omega)$ are equivalent, which means that

$$
\left\|{ }_{0} I_{x}^{\frac{\beta}{2}} p_{h}^{n}\right\|_{\frac{\beta}{2}} \leq C\left\|_{0} I_{x}^{\frac{\beta}{2}} p_{h}^{n}\right\|_{J_{L}^{\frac{\beta}{2}}}
$$

Since ${ }_{0} I_{x}^{\frac{\beta}{2}}$ is a bounded linear operator from $L^{2}(\Omega)$ to $J_{L}^{\frac{\beta}{2}}(\Omega),(3.14)$ turns out to be

$$
\left\|p_{h}^{n}\right\|^{2} \leq C\left\|q_{h}^{n}\right\|_{-\frac{\beta}{2}}\left\|p_{h}^{n}\right\|
$$

which means that

$$
\left\|p_{h}^{n}\right\| \leq C\left\|q_{h}^{n}\right\|_{-\frac{\beta}{2}} .
$$

Then

$$
\tau \sum_{n=1}^{J}\left\|p_{h}^{n}\right\|^{2} \leq \tau \sum_{n=1}^{J}\left\|q_{h}^{n}\right\|_{-\frac{\beta}{2}}^{2}
$$

Combined with (3.13), we have

$$
\max _{1 \leq n \leq J}\left\|u_{h}^{n}\right\|^{2}+\tau \sum_{n=1}^{J}\left\|q_{h}^{n}\right\|_{-\frac{\beta}{2}}^{2}+\tau \sum_{n=1}^{J}\left\|p_{h}^{n}\right\|^{2} \leq C(T, K, \beta)\left(\left\|u_{h}^{0}\right\|^{2}+\max _{1 \leq n \leq J}\left\|f^{n}\right\|^{2}\right)
$$

which completes the proof. 


\section{Convergence analysis}

Based on the conclusions presented in the above sections, we shall conduct the convergence analysis for the fully discrete expanded mixed finite element scheme. For this purpose, we borrow the elliptic projection operator $R_{h}$ which is defined as

(a) $\left(q-R_{h} q, v_{h}\right)+\left(u-R_{h} u, D v_{h}\right)=0, \quad \forall v_{h} \in V_{h}$,

(b) $\left(p-R_{h} p, \sigma_{h}\right)+\left(K\left(\theta_{0} I_{x}^{\beta}+(1-\theta)_{x} I_{1}^{\beta}\right)\left(q-R_{h} q\right), \sigma_{h}\right)=0, \quad \forall \sigma_{h} \in H_{h}$,

(c) $\left(D p-D R_{h} p, w_{h}\right)=0, \quad \forall w_{h} \in W_{h}$.

The elliptic projection operator $R_{h}$ satisfies estimate properties which are given in [36], We have

$$
\begin{aligned}
& \left\|R_{h} u-u\right\| \leq C h^{\min \left\{k+1, s-1+\frac{\beta}{2}\right\}}\|u\|_{s}, \\
& \left\|R_{h} p-p\right\| \leq C h^{\min \left\{k+1+\frac{\beta}{2}, s-1+\frac{\beta}{2}\right\}}\|u\|_{s}, \\
& \left\|R_{h} q-q\right\|_{-\frac{\beta}{2}} \leq C h^{\min \left\{k+1+\frac{\beta}{2}, s-1+\frac{\beta}{2}\right\}}\|u\|_{s} .
\end{aligned}
$$

We let $t=t^{n}, v=v_{h}, \sigma=\sigma_{h}, w=w_{h}$ in (3.2) and subtract it from (3.4) to obtain the following error equation:
(a) $\left(q_{h}^{n}-q^{n}, v_{h}\right)+\left(u_{h}^{n}-u^{n}, D v_{h}\right)=0, \quad \forall v_{h} \in V_{h}$,
(b) $\left(p_{h}^{n}-p^{n}, \sigma_{h}\right)+\left(K\left(\theta_{0} I_{x}^{\beta}+(1-\theta)_{x} I_{1}^{\beta}\right)\left(q_{h}^{n}-q^{n}\right), \sigma_{h}\right)=0, \quad \forall \sigma_{h} \in H_{h}$,
(c) $\left(\frac{u_{h}^{n}-u_{h}^{n-1}}{\tau}-u_{t}^{n}, w_{h}\right)+\left(D\left(p_{h}^{n}-p^{n}\right), w_{h}\right)=0, \quad \forall w_{h} \in W_{h}$,
(d) $u_{h}^{0}=R_{h} u^{0}$.

For compact expression, we denote

$$
\begin{array}{ll}
\xi^{n}=q_{h}^{n}-R_{h} q^{n}, & \varphi^{n}=R_{h} q^{n}-q^{n}, \\
\eta^{n}=p_{h}^{n}-R_{h} p^{n}, & \phi^{n}=R_{h} p^{n}-p^{n}, \\
\theta^{n}=u_{h}^{n}-R_{h} u^{n}, & \rho^{n}=R_{h} u^{n}-u^{n} .
\end{array}
$$

Then we combine with the elliptic projection (4.1) to rewrite the error equation as follows:
(a) $\left(\xi^{n}, v_{h}\right)+\left(\theta^{n}, D v_{h}\right)=0, \quad \forall v_{h} \in V_{h}$,
(b) $\left(\eta^{n}, \sigma_{h}\right)+\left(K\left(\theta_{0} I_{x}^{\beta}+(1-\theta)_{x} I_{1}^{\beta}\right) \xi^{n}, \sigma_{h}\right)=0, \quad \forall \sigma_{h} \in H_{h}$,
(c) $\left(\frac{\theta^{n}-\theta^{n-1}}{\tau}, w_{h}\right)+\left(\frac{R_{h} u^{n}-R_{h} u^{n-1}}{\tau}-u_{t}^{n}, w_{h}\right)+\left(D \eta^{n}, w_{h}\right)=0$,
$\forall w_{h} \in W_{h}$,
(d) $u_{h}^{0}=R_{h} u^{0}$.

Now we shall derive the error analysis for $u_{h}^{n}-u^{n}, q_{h}^{n}-q^{n}, p_{h}^{n}-p^{n}$ in the following theorem. 
Theorem 4.1 Assume that $u \in H^{2}\left(0, T ; L^{2}(\Omega)\right) \cap H^{1}\left(0, T ; H^{s}(\Omega)\right)(s \geq 1)$. Then there exists a constant $C>0$ such that

$$
\begin{aligned}
& \max _{1 \leq n \leq J}\left\|u_{h}^{n}-u^{n}\right\|^{2}+\tau \sum_{n=1}^{J}\left\|q_{h}^{n}-q^{n}\right\|_{-\frac{\beta}{2}}^{2}+\tau \sum_{n=1}^{J}\left\|p_{h}^{n}-p^{n}\right\|^{2} \\
& \quad \leq C_{0} h^{2 \min \left\{k+1, s-1+\frac{\beta}{2}\right\}}+C_{1} \tau^{2} .
\end{aligned}
$$

Here

$$
\begin{aligned}
& C_{0}=C_{0}\left(\left\|u_{t}\right\|_{L^{2}\left(0, T ; H^{s}(\Omega)\right)}^{2},\left\|u^{0}\right\|_{L^{\infty}\left(0, T ; H^{s}(\Omega)\right)}^{2}\right), \\
& C_{1}=C_{1}\left(\left\|u_{t t}\right\|_{L^{2}\left(0, T ; H^{s}(\Omega)\right)}\right) .
\end{aligned}
$$

Proof Choose $v_{h}=\eta^{n}, \sigma_{h}=\xi^{n}, w_{h}=\theta^{n}$ in (4.3) to obtain
(a) $\left(\xi^{n}, \eta^{n}\right)+\left(\theta^{n}, D \eta^{n}\right)=0$,
(b) $\left(\eta^{n}, \xi^{n}\right)+\left(K\left(\theta_{0} I_{x}^{\beta}+(1-\theta)_{x} I_{1}^{\beta}\right) \xi^{n}, \xi^{n}\right)=0$,
(c) $\left(\frac{\theta^{n}-\theta^{n-1}}{\tau}, \theta^{n}\right)+\left(\frac{R_{h} u^{n}-R_{h} u^{n-1}}{\tau}-u_{t}^{n}, \theta^{n}\right)+\left(D \eta^{n}, \theta^{n}\right)=0$.

To estimate $\theta^{n}, \xi^{n}$ and $\eta^{n}$ we use (4.4)(c). For (4.4)(c) notice

$$
\left(\frac{\theta^{n}-\theta^{n-1}}{\tau}, \theta^{n}\right) \geq \frac{\left(\left\|\theta^{n}\right\|^{2}-\left\|\theta^{n-1}\right\|^{2}\right)}{2 \tau}
$$

and

$$
\left(\theta^{n}, D \eta^{n}\right)=\left(K\left(\theta_{0} I_{x}^{\beta}+(1-\theta)_{x} I_{1}^{\beta}\right) \xi^{n}, \xi^{n}\right)=K \cos \left(\frac{\beta \pi}{2}\right)\left\|\xi^{n}\right\|_{-\frac{\beta}{2}}^{2} \geq 0
$$

to derive

$$
\frac{1}{2 \tau}\left(\left\|\theta^{n}\right\|^{2}-\left\|\theta^{n-1}\right\|^{2}\right) \leq\left|\left(\frac{R_{h} u^{n}-R_{h} u^{n-1}}{\tau}-u_{t}^{n}, \theta^{n}\right)\right| .
$$

The right hand side of (4.5) is recast

$$
\begin{aligned}
& \left(\frac{R_{h} u^{n}-R_{h} u^{n-1}}{\tau}-u_{t}^{n}, \theta^{n}\right) \\
& \quad=\left(\frac{R_{h} u^{n}-u^{n}-\left(R_{h} u^{n-1}-u^{n-1}\right)}{\tau}+\frac{u^{n}-u^{n-1}}{\tau}-u_{t}^{n}, \theta^{n}\right) \\
& \quad=\left(\frac{\rho^{n}-\rho^{n-1}}{\tau}+\frac{u^{n}-u^{n-1}}{\tau}-u_{t}^{n}, \theta^{n}\right)
\end{aligned}
$$

and estimated term by term as follows:

$$
\left(\frac{\rho^{n}-\rho^{n-1}}{\tau}, \theta^{n}\right) \leq\left\|\frac{\rho^{n}-\rho^{n-1}}{\tau}\right\|\left\|\theta^{n}\right\|
$$


Yuan and Chen Advances in Difference Equations （2018） 2018:34

Page 11 of 15

$$
\begin{aligned}
&= \frac{1}{\tau}\left\|\int_{t_{n-1}}^{t_{n}} \rho_{r}(r) d r\right\|\left\|\theta^{n}\right\| \\
& \leq \frac{1}{\tau} \tau^{\frac{1}{2}}\left(\int_{t_{n-1}}^{t_{n}}\left\|\rho_{r}(r)\right\|^{2} d r\right)^{\frac{1}{2}}\left\|\theta^{n}\right\| \\
& \leq \frac{1}{\tau}\left(\int_{t_{n-1}}^{t_{n}}\left\|\rho_{r}(r)\right\|^{2} d r+\tau\left\|\theta^{n}\right\|^{2}\right) \\
&=\frac{1}{\tau} \int_{t_{n-1}}^{t_{n}}\left\|\rho_{r}(r)\right\|^{2} d r+\left\|\theta^{n}\right\|^{2}, \\
&\left(\frac{\left.u^{n}-u^{n-1}-u_{t}^{n}, \theta^{n}\right)}{\tau} \leq\left\|\frac{u^{n}-u^{n-1}}{\tau}-u_{t}^{n}\right\|\left\|\theta^{n}\right\|\right. \\
&=\frac{1}{\tau}\left\|\int_{t_{n-1}}^{t_{n}}\left(r-t_{n-1}\right) u_{t t}(r) d r\right\| \theta^{n} \| \\
& \leq \frac{1}{\tau}\left(\int_{0}^{1} \tau^{3} \int_{t_{n-1}}^{t_{n}} u_{t t}^{2} d r d x\right)^{\frac{1}{2}}\left\|\theta^{n}\right\| \\
&=\tau \frac{1}{\frac{1}{2}}\left(\int_{t_{n-1}}^{t_{n}}\left\|u_{t t}\right\|^{2} d r\right)^{\frac{1}{2}}\left\|\theta^{n}\right\| \\
& \leq \int_{t_{n-1}}^{t_{n}}\left\|u_{t t}\right\|^{2} d r+\left\|\theta^{n}\right\|^{2} .
\end{aligned}
$$

Hence, the right hand side of (4.5) is bounded by

$$
\frac{1}{\tau} \int_{t_{n-1}}^{t_{n}}\left\|\rho_{r}(r)\right\|^{2} d r+\tau \int_{t_{n-1}}^{t_{n}}\left\|u_{t t}\right\|^{2} d r+2\left\|\theta^{n}\right\|^{2}
$$

Substitute (4.7) into (4.5) to give

$$
\frac{\left(\left\|\theta^{n}\right\|^{2}-\left\|\theta^{n-1}\right\|^{2}\right)}{2 \tau}+C\left\|\xi^{n}\right\|_{-\frac{\beta}{2}}^{2} \leq \frac{1}{\tau} \int_{t_{n-1}}^{t_{n}}\left\|\rho_{r}(r)\right\|^{2} d r+\tau \int_{t_{n-1}}^{t_{n}}\left\|u_{t t}\right\|^{2} d r+2\left\|\theta^{n}\right\|^{2} .
$$

Multiply two sides of this inequality by $2 \tau$ :

$$
\left\|\theta^{n}\right\|^{2}-\left\|\theta^{n-1}\right\|^{2}+2 C \tau\left\|\xi^{n}\right\|_{-\frac{\beta}{2}}^{2} \leq 2 \int_{t_{n-1}}^{t_{n}}\left\|\rho_{r}(r)\right\|^{2} d r+2 \tau^{2} \int_{t_{n-1}}^{t_{n}}\left\|u_{t t}\right\|^{2} d r+4 \tau\left\|\theta^{n}\right\|^{2} .
$$

We add all the terms from $n=1$ to $n=J$ and notice $\theta^{0}=u_{h}^{0}-R_{h} u^{0}=0$ to get

$$
\left\|\theta^{J}\right\|^{2}+2 C \tau \sum_{n=1}^{J}\left\|\xi^{n}\right\|_{-\frac{\beta}{2}}^{2} \leq 2 \int_{0}^{T}\left\|\rho_{r}(r)\right\|^{2} d r+2 \tau^{2} \int_{0}^{T}\left\|u_{t t}\right\|^{2} d r+4 \tau \sum_{n=1}^{J}\left\|\theta^{n}\right\|^{2} .
$$

Apply the discrete Gronwall inequality to derive

$$
\begin{aligned}
& \left\|\theta^{J}\right\|^{2}+2 C \tau \sum_{n=1}^{J}\left\|\xi^{n}\right\|_{-\frac{\beta}{2}}^{2} \\
& \quad \leq C\left(\int_{0}^{T}\left\|\rho_{r}(r)\right\|^{2} d r+\tau^{2} \int_{0}^{T}\left\|u_{t t}\right\|^{2} d r\right)
\end{aligned}
$$




$$
\begin{aligned}
= & C h^{2 \min \left\{k+1, s-1+\frac{\beta}{2}\right\}} \int_{0}^{T}\left\|u_{t}\right\|_{s}^{2} d r \\
& +C \tau^{2} \int_{0}^{T}\left\|u_{t t}\right\|^{2} d r \\
= & C h^{2 \min \left\{k+1, s-1+\frac{\beta}{2}\right\}}\left\|u_{t}\right\|_{L^{2}\left(0, T ; H^{s}(\Omega)\right)}^{2} \\
& +C \tau^{2}\left\|u_{t t}\right\|_{L^{2}\left(0, T ; L^{2}(\Omega)\right)}^{2} .
\end{aligned}
$$

It remains to estimate $\eta^{n}$. Notice that the spaces $V_{h}$ and $H_{h}$ are the same, we choose $v_{h}=\eta^{n}, \sigma_{h}=\eta^{n}, w_{h}=\theta^{n}$ in (4.3). Similar to the proof of Theorem 3.2, we can get

$$
\begin{aligned}
\tau \sum_{n=1}^{J}\left\|\eta^{n}\right\|^{2} \leq & C \tau \sum_{n=1}^{J}\left\|\xi^{n}\right\|_{-\frac{\beta}{2}}^{2} \\
\leq & C h^{2 \min \left\{k+1, s-1+\frac{\beta}{2}\right\}}\left\|u_{t}\right\|_{L^{2}\left(0, T ; H^{s}(\Omega)\right)}^{2} \\
& +C \tau^{2}\left\|u_{t t}\right\|_{L^{2}\left(0, T ; L^{2}(\Omega)\right)}^{2}
\end{aligned}
$$

Hence, we can obtain

$$
\begin{aligned}
& \max _{1 \leq n \leq J}\left\|u_{h}^{n}-u^{n}\right\|^{2}+\tau \sum_{n=1}^{J}\left\|q_{h}^{n}-q^{n}\right\|_{-\frac{\beta}{2}}^{2}+\tau \sum_{n=1}^{J}\left\|p_{h}^{n}-p^{n}\right\|^{2} \\
& \quad \leq C_{0} h^{2 \min \left\{k+1, s-1+\frac{\beta}{2}\right\}}+C_{1} \tau^{2},
\end{aligned}
$$

which completes the proof.

From Theorem 4.1 we see the estimate for $q$ is optimal and the estimate for $u$ is optimal when $k+1 \leq s-1+\frac{\beta}{2}$.

\section{Numerical experiments}

In this section we perform two numerical experiments to verify the theoretical convergence results. Here we show computations with different $\beta$ to test $\beta$-dependent convergence rates for the lowest-order $(k=0)$ Raviart-Thomas mixed finite element procedure.

Example 1 Let $K=1, \theta=\frac{1}{2}, T=1$. The analytic and smooth solution is prescribed to be $u(x)=x^{2}(1-x)^{2} e^{t} \in H^{2+\gamma}(\Omega)$, and the source term $f=e^{t}\left(x^{2}+(1-x)^{2}\right)+e^{t}\left(\frac{-12\left[x^{2+\beta}+(1-x)^{2+\beta}\right]}{\Gamma(3+\beta)}+\right.$ $\left.\frac{6\left[(1-x)^{1+\beta}+x^{1+\beta}\right]}{\Gamma(2+\beta)}-\frac{x^{\beta}+(1-x)^{\beta}}{\Gamma(1+\beta)}\right) \in H^{\beta+\gamma}(\Omega) . \gamma \in\left[0, \frac{1}{2}\right)$ can be selected as close to $\frac{1}{2}$ as possible $[36,40]$. We denote $\left\|p-p_{h}\right\|_{L^{2}\left(0, T ; H^{1}(\Omega)\right)},\left\|q-q_{h}\right\|_{L^{2}\left(0, T ; H^{\frac{-\beta}{2}}(\Omega)\right)}$ by $\left\|p-p_{h}\right\| \|$ and $\left\|q-q_{h}\right\| \|$, respectively, in Table 1 to Table 4.

The numerical results for Example 1 are presented in Table 1, Table 2 and Figure 1.

Table 1 shows that the convergence rate for $u$ is 1 under the $L^{2}$-norm, which exactly obey the prediction of Theorem 4.1. The convergence rates for $q$ and $p$ are $1+\gamma+\frac{\beta}{2}$ under the respective norms, which are almost close to the prediction of Theorem 4.1.

Table 2 tests that the convergence rate in time. The numerical results show that the convergence rate for $u, p, q$ in time is 1 which is consistent with the theoretical prediction by Theorem 4.1 . 
Table 1 Numerical results for Example 1 with $\tau=2^{-12}$

\begin{tabular}{lllllll}
\hline $\boldsymbol{\beta}$ & $\boldsymbol{h}$ & $\mathbf{1 / 1 6}$ & $\mathbf{1 / 3 2}$ & $\mathbf{1 / 6 4}$ & $\mathbf{1 / 1 2 8}$ & Order \\
\hline $1 / 4$ & $\left\|u-u_{h}\right\|$ & $9.50 \mathrm{e}-03$ & $4.78 \mathrm{e}-03$ & $2.40 \mathrm{e}-03$ & $1.20 \mathrm{e}-03$ & 1.00 \\
& $\left\|p-p_{h}\right\|$ & $3.33 \mathrm{e}-03$ & $9.62 \mathrm{e}-04$ & $2.81 \mathrm{e}-04$ & $8.20 \mathrm{e}-05$ & 1.78 \\
& $\left\|q-q_{h}\right\|$ & $4.44 \mathrm{e}-03$ & $1.43 \mathrm{e}-03$ & $4.62 \mathrm{e}-04$ & $1.50 \mathrm{e}-04$ & 1.60 \\
$1 / 2$ & $\left\|u-u_{h}\right\|$ & $9.48 \mathrm{e}-03$ & $4.77 \mathrm{e}-03$ & $2.39 \mathrm{e}-03$ & $1.20 \mathrm{e}-03$ & 1.00 \\
& $\left\|p-p_{h}\right\|$ & $1.43 \mathrm{e}-03$ & $3.94 \mathrm{e}-04$ & $1.07 \mathrm{e}-04$ & $2.79 \mathrm{e}-05$ & 1.92 \\
& $\left\|q-q_{h}\right\|$ & $3.90 \mathrm{e}-03$ & $1.15 \mathrm{e}-03$ & $3.45 \mathrm{e}-04$ & $1.05 \mathrm{e}-04$ & 1.73 \\
\multirow{3}{*}{$2 / 3$} & $\left\|u-u_{h}\right\|$ & $1.48 \mathrm{e}-02$ & $7.53 \mathrm{e}-03$ & $3.78 \mathrm{e}-03$ & $1.89 \mathrm{e}-03$ & 1.00 \\
& $\left\|p-p_{h}\right\|$ & $6.15 \mathrm{e}-04$ & $1.69 \mathrm{e}-04$ & $4.54 \mathrm{e}-05$ & $1.19 \mathrm{e}-05$ & 1.94 \\
& $\left\|q-q_{h}\right\|$ & $3.98 \mathrm{e}-03$ & $1.09 \mathrm{e}-03$ & $3.02 \mathrm{e}-04$ & $8.37 \mathrm{e}-05$ & 1.84 \\
\hline
\end{tabular}

Table 2 Numerical results for Example 1 with $h=2^{-8}$ and $\beta=\frac{2}{3}$

\begin{tabular}{llllll}
\hline $\boldsymbol{\tau}$ & $\mathbf{1 / 8}$ & $\mathbf{1 / 1 6}$ & $\mathbf{1 / 3 2}$ & $\mathbf{1 / 6 4}$ & Order \\
\hline$\left\|u-u_{h}\right\|$ & $3.21 \mathrm{e}-03$ & $1.25 \mathrm{e}-03$ & $5.72 \mathrm{e}-04$ & $2.83 \mathrm{e}-04$ & 1.00 \\
$\left\|p-p_{h}\right\|$ & $1.23 \mathrm{e}-03$ & $6.36 \mathrm{e}-04$ & $3.21 \mathrm{e}-04$ & $1.60 \mathrm{e}-04$ & 1.00 \\
$\left\|q-q_{h}\right\|$ & $4.12 \mathrm{e}-03$ & $2.01 \mathrm{e}-03$ & $1.01 \mathrm{e}-03$ & $5.11 \mathrm{e}-04$ & 0.98 \\
\hline
\end{tabular}

Table 3 Numerical results for Example 2 with $\tau=2^{-10}$

\begin{tabular}{lllllll}
\hline $\boldsymbol{\beta}$ & $\boldsymbol{h}$ & $\mathbf{1 / 1 6}$ & $\mathbf{1 / 3 2}$ & $\mathbf{1 / 6 4}$ & $\mathbf{1 / 1 2 8}$ & Order \\
\hline $1 / 4$ & $\left\|u-u_{h}\right\|$ & $3.62 \mathrm{e}-02$ & $1.91 \mathrm{e}-02$ & $9.77 \mathrm{e}-03$ & $4.95 \mathrm{e}-03$ & 0.99 \\
& $\left\|p-p_{h}\right\|$ & $6.51 \mathrm{e}-02$ & $3.84 \mathrm{e}-02$ & $2.28 \mathrm{e}-02$ & $1.35 \mathrm{e}-02$ & 0.75 \\
& $\left\|q-q_{h}\right\|$ & $1.13 \mathrm{e}-02$ & $7.26 \mathrm{e}-03$ & $4.69 \mathrm{e}-03$ & $3.04 \mathrm{e}-03$ & 0.63 \\
$1 / 2$ & $\left\|u-u_{h}\right\|$ & $3.62 \mathrm{e}-02$ & $1.91 \mathrm{e}-02$ & $9.77 \mathrm{e}-03$ & $4.95 \mathrm{e}-03$ & 0.99 \\
& $\left\|p-p_{h}\right\|$ & $1.84 \mathrm{e}-02$ & $9.04 \mathrm{e}-03$ & $4.48 \mathrm{e}-03$ & $2.23 \mathrm{e}-03$ & 1.00 \\
& $\left\|q-q_{h}\right\|$ & $1.03 \mathrm{e}-02$ & $6.11 \mathrm{e}-03$ & $3.62 \mathrm{e}-03$ & $2.15 \mathrm{e}-03$ & 0.75 \\
$2 / 3$ & $\left\|u-u_{h}\right\|$ & $3.62 \mathrm{e}-02$ & $1.91 \mathrm{e}-02$ & $9.77 \mathrm{e}-03$ & $4.95 \mathrm{e}-03$ & 0.99 \\
& $\left\|p-p_{h}\right\|$ & $6.86 \mathrm{e}-03$ & $2.99 \mathrm{e}-03$ & $1.31 \mathrm{e}-03$ & $5.82 \mathrm{e}-04$ & 1.15 \\
& $\left\|q-q_{h}\right\|$ & $8.18 \mathrm{e}-03$ & $4.59 \mathrm{e}-03$ & $2.58 \mathrm{e}-03$ & $1.46 \mathrm{e}-03$ & 0.82 \\
\hline
\end{tabular}

Table 4 Numerical results for Example 2 with $h=2^{-8}$ and $\beta=\frac{2}{3}$

\begin{tabular}{llllll}
\hline $\boldsymbol{\tau}$ & $\mathbf{1 / 8}$ & $\mathbf{1 / 1 6}$ & $\mathbf{1 / 3 2}$ & $\mathbf{1 / 6 4}$ & Order \\
\hline$\left\|u-u_{h}\right\|$ & $3.75 \mathrm{e}-02$ & $1.10 \mathrm{e}-02$ & $3.58 \mathrm{e}-03$ & $1.44 \mathrm{e}-03$ & 1.30 \\
$\left\|p-p_{h}\right\|$ & $9.00 \mathrm{e}-03$ & $3.20 \mathrm{e}-03$ & $1.50 \mathrm{e}-03$ & $7.42 \mathrm{e}-04$ & 1.02 \\
$\left\|q-q_{h}\right\|$ & $1.95 \mathrm{e}-03$ & $9.49 \mathrm{e}-03$ & $4.71 \mathrm{e}-03$ & $2.35 \mathrm{e}-03$ & 1.00 \\
\hline
\end{tabular}

Figure 1 The sketch of Example 1 about errors and degrees of freedom with $\beta=\frac{2}{3}$.

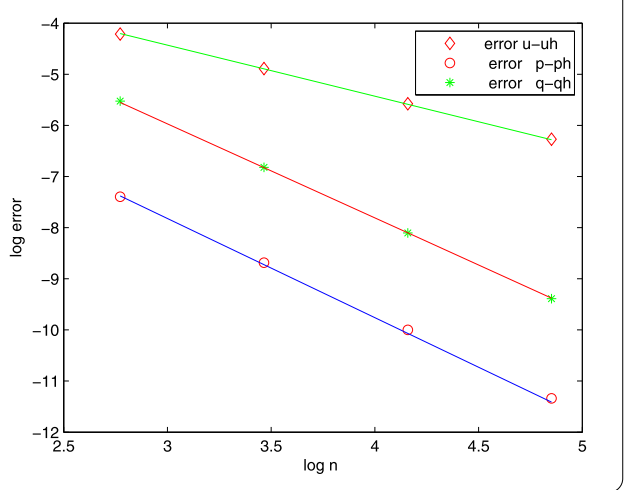

Example 2 Let $K=1, \theta=\frac{1}{2}, T=1$. The analytic and smooth solution is prescribed to be $u(x)=x(1-x) e^{t} \in H^{1+\gamma}(\Omega)$, and the source term $f=x(1-x) e^{t}+e^{t}\left(-\frac{x^{\beta-1}+(1-x)^{\beta-1}}{2 \Gamma(\beta)}+\right.$ $\left.\frac{x^{\beta}+(1-x)^{\beta}}{\Gamma(1+\beta)}\right) \in H^{\beta-1+\gamma}(\Omega) \cdot \gamma$ is defined as in Example 1 . 


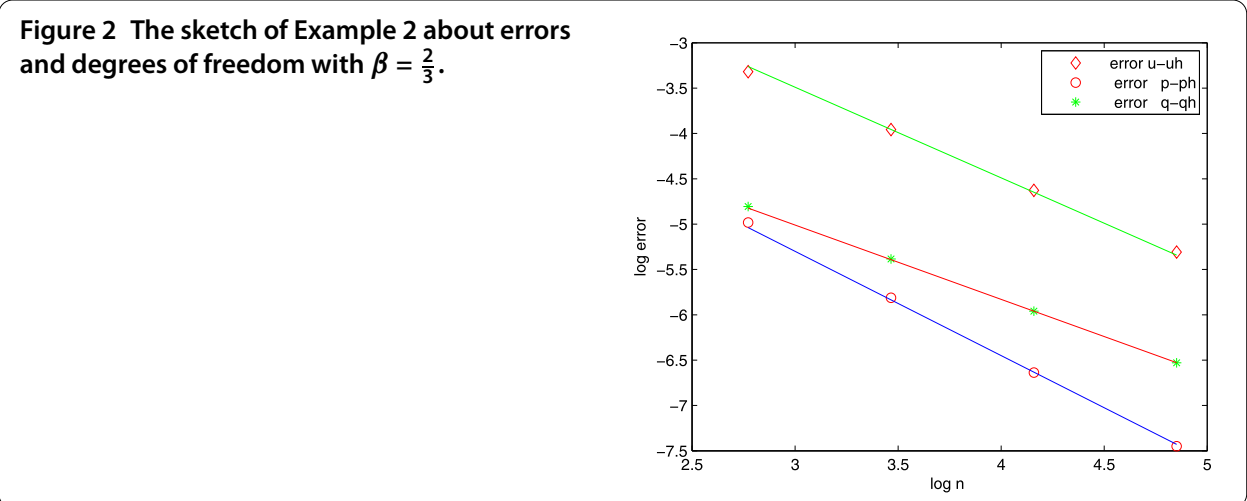

The numerical results are presented in Table 3, Table 4 and Figure 2.

Table 3 shows that the convergence rate for $u$ is 1 under the $L^{2}$-norm, which exactly obey the prediction of Theorem 4.1. The convergence rates for $q$ and $p$ are $\gamma+\frac{\beta}{2}$ under the respective norms, which are almost close to the prediction of Theorem 4.1.

Table 4 tests that the convergence rate in time. The numerical results show that the convergence rate for $u, p, q$ in time is 1 which is consistent with the theoretical prediction by Theorem 4.1 .

\section{Concluding remarks}

In this work, we establish the saddle-point variational formulation for a two-sided timedependent fractional diffusion problem over $H^{1}(\Omega) \times H^{-\frac{\beta}{2}}(\Omega) \times L^{2}(\Omega)$ and develop its fully discrete expanded mixed finite element procedure, which approximates optimally the unknown function $u$, its derivative $q$ and the fractional flux $p$. We find that the advantages of the mixed procedure at least are: (1) it can preserve the locally conservative property and can describe realistic fractional diffusive processes; (2) it is easily implemented since the standard finite element spaces are used.

\section{Acknowledgements}

This work is supported by NSF of China under Grants 11471196, 20142RB01849 and by NSF of Shandong province under Grant ZR2016JL004.

Competing interests

The authors declare that they have no competing interests.

Authors' contributions

All authors contributed equally to this work. All authors read and approved the final manuscript.

\section{Publisher's Note}

Springer Nature remains neutral with regard to jurisdictional claims in published maps and institutional affiliations.

Received: 16 October 2017 Accepted: 7 January 2018 Published online: 23 January 2018

\section{References}

1. Mainardi, F: Fractional calculus. In: Carpinteri, A, Mainardi, F (eds.) Fractals and Fractional Calculus in Continuum Mechanics. Springer, New York (1997)

2. Shlesinger, MF, West, BJ, Klafter, J: Lévy dynamics of enhanced diffusion: application to turbulence. Phys. Rev. Lett. 58(11), 1100-1103 (1987)

3. Zaslavsky, GM, Stevens, D, Weitzner, H: Self-similar transport in incomplete chaos. Phys. Rev. E 48(3), 1683-1694 (1993)

4. Sun, $\mathrm{H}, \mathrm{Hao}, \mathrm{X}, \mathrm{Zhang}, \mathrm{Y}$, Beleanu, D: Relaxation and diffusion models with non-singular kernels. Phys. A, Stat. Mech. Appl. 468, 590-596 (2017)

5. Beleanu, D, Jajarmi, A, Hajipour, M: A new formulation of the fractional optimal control problems involving Mittag-Leffler nonsingular kernel. J. Optim. Theory Appl. 175(3), 718-737 (2017) 
6. Caputo, M, Fabrizio, M: A new definition of fractional derivative without singular kernel. Prog. Fract. Differ. Appl. 1, 73-85 (2015)

7. Singh, J, Kumar, D, Baleanu, D: On the analysis of chemical kinetics system pertaining to a fractional derivative with Mittag-Leffler type kernel. Chaos 27 (2017). https://doi.org/10.2298/TSCI170129096K

8. Kumar, D, Singh, J, Baleanu, D: Modified Kawahara equation within a fractional derivative with non-singular kernel. Therm. Sci. (2017). https://doi.org/10.1063/1.4995032

9. Kumar, D, Singh, J, Baleanu, D: A new fractional model for convective straight fins with temperature-dependent thermal conductivity. Therm. Sci. (2017). https://doi.org/10.2298/TSCl170129096K

10. Beleanu, D, Jajarmi, A, Asad, J, Blaszczyk, T: The motion of a bead sliding on a wire in fractional sense. Acta Phys. Pol. A $131(6), 1561-1564(2017)$

11. Cui, M: Compact finite difference method for the fractional diffusion equation. J. Comput. Phys. 228, 7792-7804 (2009)

12. Meerschaert, MM, Scheffler, HP, Tadjeran, C: Finite difference methods for two dimensional fractional dispersion equation. J. Comput. Phys. 211, 249-261 (2006)

13. Li, C, Zeng, F, Liu, F: Spectral approximations to the fractional integral and derivative. Fract. Calc. Appl. Anal. 15, 383-406 (2012)

14. Wang, H, Basu, TS: A fast finite difference method for two-dimensional space-fractional diffusion equations. SIAM J. Sci. Comput. 34, 2444-2458 (2012)

15. Liu, Q, Liu, F, Turner, I, Anh, V: Finite element approximation for the modified anomalous subdiffusion process. Appl. Math. Model. 35, 4103-4116 (2011)

16. Zhang, H, Liu, F, Anh, V: Garlerkin finite element approximations of symmetric space fractional partial differential equations. Appl. Math. Comput. 217, 2534-2545 (2010)

17. Kumar, D, Singh, J, Baleanu, D: A new numerical algorithm for fractional Fitzhugh-Nagumo equation arising in transmission of nerve impulses. Nonlinear Dyn. (2017). https://doi.org/10.1007/s11071-017-3870-x

18. Kumar, D, Agarwal, RP, Singh, J: A modified numerical scheme and convergence analysis for fractional model of Lienard's equation. J. Comput. Appl. Math. (2017). https://doi.org/10.1016/..cam.2017.03.011

19. Hajipour, M, Jajarmi, A, Baleanu, D: An efficient non-standard finite difference scheme for a class of fractional chaotic systems. J. Comput. Nonlinear Dyn. 13(2) (2017). https://doi.org/10.1115/1.4038444

20. Wu, G, Baleanu, D, Deng, Z, Zeng, S: Lattice fractional diffusion equation in terms of a Riesz-Caputo difference. Physica A 438, 335-339 (2015)

21. Wu, G, Baleanu, D, Xie, H: Riesz Riemann-Liouville difference on discrete domains. Chaos 26, 084308 (2016)

22. Ervin, VJ, Heuer, N, Roop, JP: Numerical approximation of a time dependent, nonlinear, space-fractional diffusion equation. SIAM J. Numer. Anal. 45, 572-591 (2007)

23. Ervin, VJ, Roop, JP: Variational formulation for the stationary fractional advection dispersion equation. Numer. Methods Partial Differ. Equ. 22, 558-576 (2005)

24. Ervin, VJ, Roop, JP: Variational solution of fractional advection dispersion equations on bounded domains in $R^{d}$. Numer. Methods Partial Differ. Equ. 23, 256-281 (2007)

25. Deng, W, Hesthaven, JS: Discontinuous Galerkin methods for fractional diffusion equations. Math. Model. Anal. 47(6), 1845-1864 (2013)

26. Li, Y, Chen, H: A mixed-type Galerkin variational formulation and fast algorithms for variable-coefficient fractional diffusion equations. Math. Methods Appl. Sci. 40(4), 5016-5034 (2017)

27. Jia, L, Chen, H: Mixed-type Galerkin variational priciple and numerical simulation for a generalized nonlocal elastic model. J. Sci. Comput. 71(2), 660-681 (2017)

28. Raviart, PA, Thomas, JM: A mixed finite element methods for 2 nd order elliptic problems. In: Mathmatical Aspects of the Finite Element Method. Lecture Notes in Maths., vol. 606, pp. 292-315. Springer, Berlin (1977)

29. Liu, Y, Du, Y, Li, H, Li, J, He, S: A two-grid mixed finite element method for a nonlinear fourth-order reaction-diffusion problem with time-fractional derivative. Comput. Math. Appl. 70, 2474-2492 (2015)

30. Liu, Y, Fang, Z, Li, H, He, S: A mixed finite element method for a time-fractional fourth-order partial differential equation. Appl. Math. Comput. 243, $703-717$ (2014)

31. Wang, H, Yang, D, Zhu, S: A Petrov-Galerkin finite element method for variable-coefficient fractional diffusion equations. Comput. Methods Appl. Mech. Eng. 290, 45-56 (2015)

32. Yang, S, Chen, H: Least-squared mixed variational formulation based on space decomposition for a kind of variable-coefficient fractional diffusion problems. Manuscript

33. Oldham, KB, Spanier, J: The Fractional Calculus. Academic Press, New York (1974)

34. Podlubny, I: Fractional Differential Equations. Academic Press, New York (1999)

35. Samko, S, Kilbas, A, Marichev, O: Fractional Integrals and Derivatives: Theory and Applications. Gordon \& Breach, London (1993)

36. Chen, $\mathrm{H}$, Wang, $\mathrm{H}$ : Numerical simulation for conservative fractional diffusion equations by an expanded mixed formulation. J. Comput. Appl. Math. 296, 480-498 (2016)

37. Brézzi, F, Douglas, J Jr., Fortin, M, Marini, L: Two families of mixed finite element for second-order elliptic problems. Numer. Math. 47, 217-235 (1985)

38. Brézzi, F, Fortin, M: Mixed and Hybrid Finite Element Methods. Springer, Berlin (2011)

39. Chen, Z: Expanded mixed finite element methods for linear second-order elliptic equation. Math. Model. Anal. 4, 479-499 (1998)

40. Jin, BT: Variational formulation of problems involving fractional order differential operators. Math. Comput. 84, 2665-2700 (2015) 